\title{
Mutations in dynamin 2 cause dominant Centronuclear Myopathy
}

Marc Bitoun ${ }^{1}$, Svetlana Maugenre ${ }^{1}$, Pierre-Yves Jeannet ${ }^{2}$, Emmanuelle Lacène ${ }^{1}$, Xavier Ferrer ${ }^{3}$, Pascal Laforêt ${ }^{1}$, Jean-Jacques Martin ${ }^{4}$, Jocelyn Laporte ${ }^{5}$, Hanns Lochmüller ${ }^{6}$, Alan H. Beggs ${ }^{7}$, Michel Fardeau ${ }^{1}$, Bruno Eymard ${ }^{1}$, Norma B. Romero ${ }^{1}$ and Pascale Guicheney ${ }^{1}$.

1. INSERM U582, Institute of Myology, IFR14, Groupe Hospitalier Pitié-Salpêtrière, UPMC, Paris, France.

2. Department of Pediatrics, Neuropediatric Unit, CHUV, Lausanne, Switzerland.

${ }^{3}$. Department of Neurology, CHU Haut-Lévêque, Bordeaux, France.

${ }^{4}$. Laboratory of Neuropathology, Born-Bunge Institute, Antwerp, Belgium.

5. Department of Molecular Pathology, I.G.B.M.C., CNRS/INSERM/ULP/Collège de France, Illkirch, France.

6. Friedrich Baur Institute, Department of Neurology, Ludwig Maximilians University, Munich, Germany.

7. Genetics Division, Children’s Hospital Boston, Harvard Medical School, Boston, USA.

\section{Acknowledgements}

We thank the patients and their families for their participation in this study; JP. Leroy and JL. Mandel for continuous support; E. Ralston for comments and suggestions on the manuscript; A. Rouche for assistance with immunohistochemistry, C. Lacroix for providing a DNA sample. This work was supported by the Institut National de la Santé et de la Recherche Médicale (INSERM), the Centre National de la Recherche Scientifique (CNRS), the Hôpital Universitaire de Strasbourg (HUS), the Collège de France and the Association Française contre les Myopathies (AFM). H.L. is member of the German Muscular Dystrophy Network (MD-NET, 01GM0302) supported by the German Ministry of Education and Research (BMBF, Bonn, Germany). P-Y.J. was a recipient of a fellowship from the Swiss Foundation for Research on Muscle Diseases. AHB was supported by NIH NS40828 and the Joshua Frase Foundation. M.B. was the recipient of an AFM fellowship.

\section{Corresponding author:}

Dr. Pascale Guicheney

Unité INSERM 582 
Groupe Hospitalier Pitié-Salpêtrière

47 Boulevard de l'Hôpital

\section{Paris Cedex 13}

phone: +33-1-4216 5705

fax: $\quad+33-1-42165700$

e-mail: p.guicheney@myologie.chups.jussieu.fr 
Autosomal dominant centronuclear myopathy (CNM) is a rare congenital myopathy characterized by delayed motor milestones and muscular weakness. In 11 families affected by CNM, we identified recurrent and de novo missense mutations in dynamin 2 gene (DNM2, 19p13.2), encoding a protein involved in endocytosis and membrane trafficking, actin assembly, and centrosome cohesion. The transfected mutants showed a reduced labelling in the centrosome suggesting that DNM2 mutations may cause CNM by interfering with centrosome function. 
Autosomal dominant centronuclear myopathy (CNM) is a congenital myopathy, first described in $1966^{1}$, characterized by a slowly progressive muscular weakness and wasting. The disorder involves mainly limb girdle, trunk and neck muscles, but may also affect the distal muscles. Weakness may be present during childhood or adolescence or may only become evident in the third decade of life. Some patients become wheelchair-bound in their fifties. Ptosis and limitation of eye movements are frequent. The most prominent histopathological features consist of a high frequency of centrally located nuclei in a large number of the extrafusal muscle fibres, a radial arrangement of sarcoplasmic strands around the central nuclei, and predominance and hypotrophy of type 1 fibers $^{2,3}$. The severe X-linked form of CNM, called X-linked myotubular myopathy, is caused by mutations in the MTM1 gene coding for myotubularin, a highly conserved phosphoinositide phosphatase probably involved in membrane trafficking ${ }^{4}$.

A genome-wide linkage mapping was performed in two autosomal dominant CNM families with initially 8 and 6 studied affected subjects (Family 1 and Family 2, respectively). Only three markers, D19S884, D19S865 and D19S226, gave positive lod scores in these two families. The locus was confirmed by genotyping other affected family members and a third multigenerational family (Family 3). Additional microsatellite markers were genotyped and several recombination events mapped the CNM locus on chromosome 19p13.2 in a 11.03 Mb interval between D19S1034 and D19S899 (Supplementary Table 1 and Supplementary Fig. 1).

Candidate proteins were searched using three criteria: i) expression in skeletal muscle, ii) involvement in membrane trafficking (myotubularin-like hypothesis), and iii) direct or indirect link to cytoskeleton potentially responsible for morphological abnormalities observed in CNM. Using these criteria, we focused on the DNM2 gene which encodes the ubiquitous protein dynamin 2 $(\text { DNM2 })^{5}$. Dynamins are large GTPases initially involved in membrane trafficking where they act as mechanochemical scaffolding molecules that can hydrolyse GTP to deform biological membranes $^{6}$. Among the dynamin family, the $100 \mathrm{kD}$ GTPase DNM2 has been shown to play a role in endocytosis at the plasma membrane ${ }^{6}$, and also in membrane trafficking from the trans-Golgi network $^{7}$, formation of actin stress-fibers ${ }^{8}$, actin-membrane interface assembly ${ }^{9}$ and centrosome cohesion $^{10}$.

We sequenced DNM2 exons and intron-exon boundaries in the probands of Families 1 to 3 and identified heterozygous missense mutations in both of them (Table 1 and Supplementary Fig. 2). Two were located in exon 8 changing arginine 369 to glutamine (R369Q) in Family 2 (G1106A), and the same residue to tryptophane (R369W) in Family 3 (C1105T). In Family 1, a mutation in exon 11 (C1395T) induces the change of the arginine 465 to tryptophane (R465W). Missense mutations were also identified in 8 additional CNM families with either dominant inheritance or sporadic cases. The R465W mutation was recognized in 5 additional families and the 
R369Q mutation in 2 others (Table 1). Genotyping of single nucleotide polymorphisms (SNP) surrounding R369Q and R465W mutations revealed several genetic backgrounds among patients sharing the same mutation (data not shown). A novel missense mutation, G1102A, changing glutamic acid 368 to lysine (E368K), was identified in the proband of Family 4 and not found in the parents. The paternity was confirmed, indicating that it is a de novo mutation. The R369W mutation in Family 3 also likely represents a de novo event (see Supplementary Fig. 1). All the mutations cosegregate with the disease in the 11 families and were not found in 150 healthy control subjects of European descent screened for mutations by single strand conformation polymorphism (SSCP) for mutations R465W and E368K or restriction enzyme digestion using Acil for mutations R369W and R369Q since SSCP profiles were not informative (Supplementary Fig. 2). Taken together, our results suggest that DNM2 mutations cause CNM in carrier subjects. The severity of the disease in patients harbouring the two most frequent mutations do not greatly differ, nevertheless its onset occurred earlier in some patients with the R465W mutation compared to the R369Q carriers. This will have to be confirmed in a larger series of patients.

The three mammalian dynamins share the same protein structure (Supplementary Fig. 3) with a N-terminal tripartite GTPase domain, a middle domain, a Pleckstrin Homology (PH) domain, a GTPase effector domain (GED) and a C-terminal proline rich domain (PRD). Recently, DNM2 mutations, restricted to the $\mathrm{PH}$ domain which mediates interaction with phosphoinositides, have been identified in the dominant intermediate form of Charcot-Marie-Tooth disease type B (DICMTB $)^{11}$. It is noteworthy that in CNM, the four mutations, affecting three highly conserved residues (Supplementary Fig. 3), were all restricted to the middle domain. The middle domain is involved in self-assembly of the molecule ${ }^{12}$, and the GTPase activity induces a conformational change of this domain associated with a constriction of lipidic structures ${ }^{13}$. Moreover, the middle domain was shown to be essential for the centrosomal localization of DNM2 ${ }^{10}$.

In order to explore the capability of DNM2 mutants to be located to the centrosome, we prepared GFP-chimera using wild-type-, R369W- and R465W-DNM2 constructs. The wild-typeDNM2-GFP was clearly detected in the centrosome in transfected human fibroblasts. In contrast, the fluorescence associated with centrosome was markedly reduced in the fibroblasts transfected by the GFP-DNM2 mutants and it was even nearly absent in about half of the R465W-DNM2 transfected fibroblasts (Fig. 1a). In addition, similar level of DNM2 was observed in fibroblasts from controls and R465W CNM patients studied both by cell immunolabelling and by Western blotting (Fig. 1b,c), suggesting that the mutant protein is relatively stable. Based on these results, we can hypothesize that mutations may hinder either the transport of the DNM2 to the centrosome or its interaction with some centrosomal component(s) such as $\gamma$-tubulin ${ }^{10}$. Such phenomena may be responsible for a defect in a centrosomal function through a dominant negative effect. Nevertheless, 
we cannot exclude that the CNM mutations may also alter other mechanisms in which DNM2 middle domain is involved.

Furthermore, immunohistochemical analysis with the polyclonal DNM2 antibody on muscle biopsies of 5 affected individuals and 4 controls showed no clear difference in intensity of labelling, even if there were some mild changes in its distribution most likely due to the particular rearrangement of the intermyofibrillar network (data not shown). In addition, the microtubular network visualized through $\alpha$-tubulin labelling appeared correctly organized in CNM fibroblasts (Fig. 1d). Nevertheless, if microtubule nucleation is not likely impaired in CNM fibroblasts, the situation could differ in skeletal muscle as during differentiation, centrosomes disappear and the Microtubule Organizing Center (MTOC) is redistributed near to the nuclear membrane and in some new cytoplasmic sites of nucleation ${ }^{14}$. Concurrently, the microtubular network is rearranged longitudinally along the axis of differentiated myotubes. It remains to be determined whether DNM2 plays a role in this reorganization in muscle and in the positioning of nuclei ${ }^{15}$.

To sum up, our results represent the first report of a gene responsible for dominant CNM. We identified several DNM2 missense mutations in the middle domain of the protein resulting in an altered centrosomal localization of DNM2 in vitro. Considering the broad range of the cellular mechanisms in which DNM2 is involved, our study opens new avenues for better understanding the pathophysiology of the centronuclear myopathies. 


\section{REFERENCES}

1. Spiro, A. J., Shy, G. M. \& Gonatas, N. K. Arch. Neurol. 14, 1-14 (1966).

2. Jeannet, P-Y. et al. Neurology 62, 1484-1490 (2004).

3. Fardeau, M. \& Tomé, F. M. In: Engel AG, Franzini-Armstrong C, eds. Myology $2^{\text {nd }} e d$. McGrawHill, inc, 1500-1504 (1994).

4. Laporte, J., Bedez, F., Bolino, A. \& Mandel, J-L. Hum. Mol. Genet. 12, R285-R292 (2003).

5. Cook, T. A., Urrutia, R. \& McNiven, M. A. Proc. Natl. Acad. Sci. U S A 91, 644-648 (1994).

6. Praefcke, G. J. \& McMahon, H. T. Nat Rev Mol Cell Biol. 5, 133-147 (2004).

7. Jones, S. M., Howell, K. E., Henley, J. R., Cao, H. \& McNiven, M. A. Science 279, 573-577 (1998).

8. Yoo, J., Jeong, M. J., Cho, H. J., Oh, E. S. \& Han, M. Y. Biochem. Biophys. Res. Commun. 328, 424-431 (2005).

9. Orth, J. D. \& McNiven, M. A. Curr. Opin. Cell Biol. 15, 31-39 (2003)

10. Thompson, H. M., Cao, H., Chen J., Euteneuer, U. \& McNiven, M. A. Nat. Cell. Biol. 6, 335342 (2004).

11. Züchner, S. et al. Nat. Genet. 37, 289-294 (2005).

12. Smirnova, E., Shurland, D. L., Newman-Smith, E. D., Pishvaee, B. \& van der Bliek, A. M. J. Biol. Chem. 274, 14942-14947 (1999).

13. Chen, Y. J., Zhang, P., Egelman, E. H. \& Hinshaw, J. E. Nat. Struct. Mol. Biol. 11, 574-575 (2004).

14. Tassin, A. M., Maro, B. \& Bornens, M. J. Cell Biol. 100, 35-46 (1985). 
15. Reinsch, S. \& Gonczy, P. J. Cell Sci. 111, 2283-2295 (1998). 


\section{LEGENDS}

Table 1: Summary of DNM2 mutations.

Nucleotide numbers are indicated relative to the first translated base (the A from the ATG initiation codon). All mutations affect CpG dinucleotides. Families 1, 2, 4, 10 and 11 have been thoroughly described in Jeannet et al. ${ }^{2}$ where they correspond to Families 1, 3, 8, 11 and 2, respectively). Family 9 from USA is a family of European origin.

Figure 1: Localization of transfected DNM2 in fibroblasts and expression of DNM2 and $\alpha$ tubulin in CNM-fibroblasts.

(a) Typical results of transfection of healthy human fibroblasts with pGFP-DNM2-wild-type (WT), pGFP-DNM2-R369W or pGFP-DNM2-R465W. Fibroblasts from two unrelated control subjects were used with similar results. While wild-type DNM2 was detected at the centrosome in most of the fibroblasts (45 cells of 48), centrosomal localization of the dynamin-mutants was altered. For the R369W mutant, fluorescence was detected at the centrosome in most of the transfected cells (35 cells of 37) but at a lower level compared to cells transfected by the wild-type form. For the R465W mutant, a reduced GFP fluorescence was only detected in about 50\% of transfected fibroblasts (15 cells of 31). Fibroblasts which have been countered were all presenting with a strong extracentrosomal fluorescence. Arrows indicate centrosomes and inserts show enlargements of the boxes placed in the centrosomal region. (b) Characteristic double immunostaining of endogenous DNM2 and $\gamma$-tubulin in control- and CNM-fibroblasts. The arrows indicate centrosomes. DNM2 was detected at the centrosomes of both control and patient fibroblasts and there were no morphological changes. Merged images (right panel) showed that DNM2 and $\gamma$-tubulin are only colocalized at the centrosome in both control and patient fibroblasts. (c) Western-blot of DNM2 in control and CNM fibroblasts. Protein level appeared unchanged in CNM-fibroblasts compared to control ones, $\alpha$ tubulin being used as loading control. (d) Microtubule network visualized in control and CNM fibroblasts using $\alpha$-tubulin immunostaining. The arrowheads show the microtubule organizing centers. There was no apparent disorganization of microtubule network in CNM fibroblasts. 


\begin{tabular}{ccccccc}
\hline Family & $\begin{array}{c}\text { Family } \\
\text { number }\end{array}$ & $\begin{array}{c}\text { Number of } \\
\text { mutation } \\
\text { carriers }\end{array}$ & Origin & $\begin{array}{c}\text { Exon } \\
\text { Nucleotide } \\
\text { change }\end{array}$ & $\begin{array}{c}\text { Amino } \\
\text { acid } \\
\text { change }\end{array}$ \\
\hline 1 & 3012 & 9 & France & 11 & C1393T & R465W \\
2 & 11451 & 14 & French Guyana & 8 & G1106A & R369Q \\
3 & 961 & 5 & France & 8 & C1105T & R369W \\
4 & 9346 & 1 & France & 8 & G1102A & E368K \\
5 & IBB/CNM1 & 4 & Belgium & 11 & C1393T & R465W \\
6 & IBB/CNM2 & 3 & Belgium & 11 & C1393T & R465W \\
7 & E/CNM3 & 4 & Germany & 11 & C1393T & R465W \\
8 & E/393 & 2 & Great Britain & 11 & C1393T & R465W \\
9 & E/703 & 4 & USA & 11 & C1393T & R465W \\
10 & 722 & 1 & France & 8 & C1105T & R369W \\
11 & 14815 & 3 & France & 8 & C1105T & R369W \\
\hline
\end{tabular}



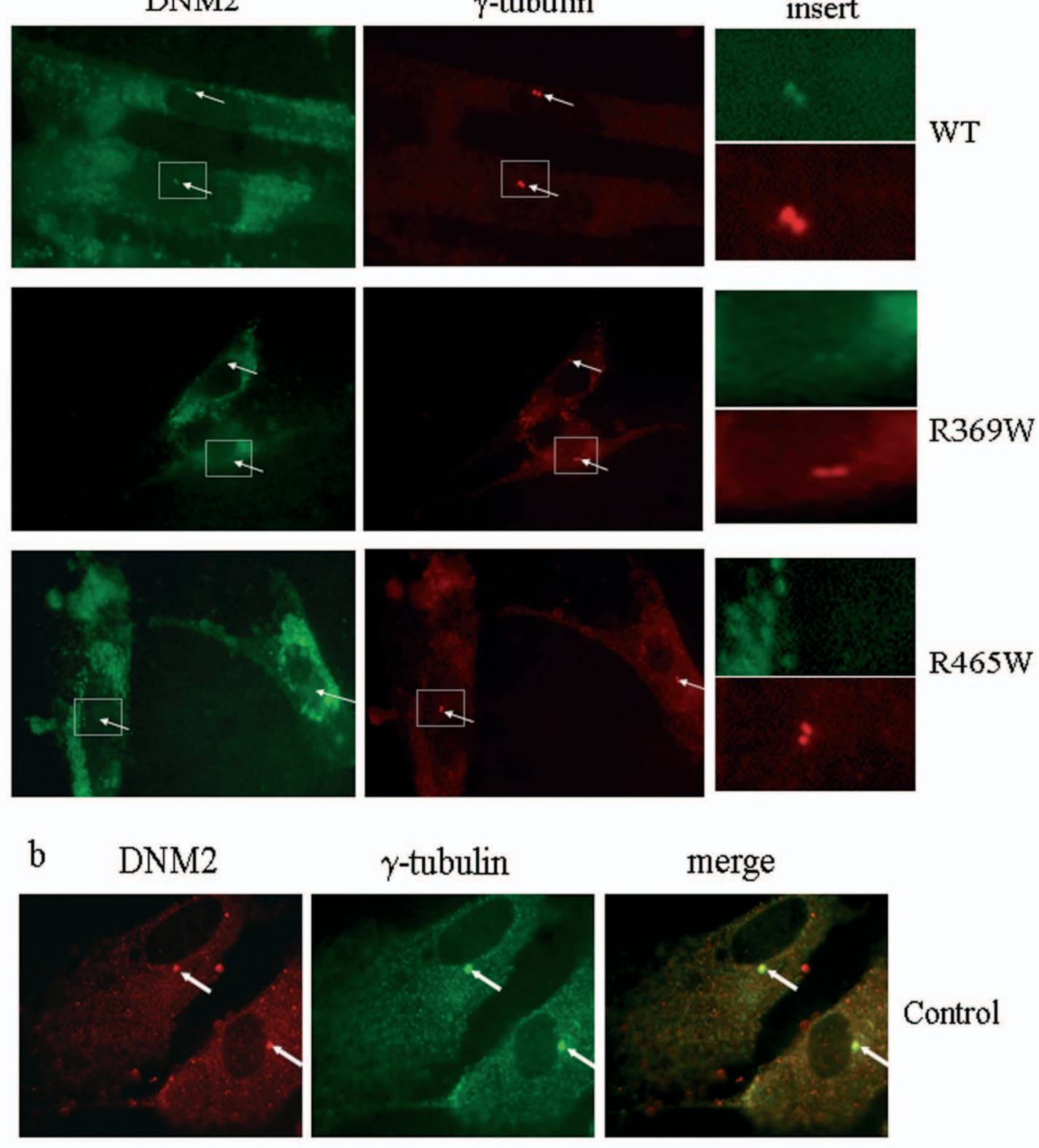

$\gamma$-tubulin

merge
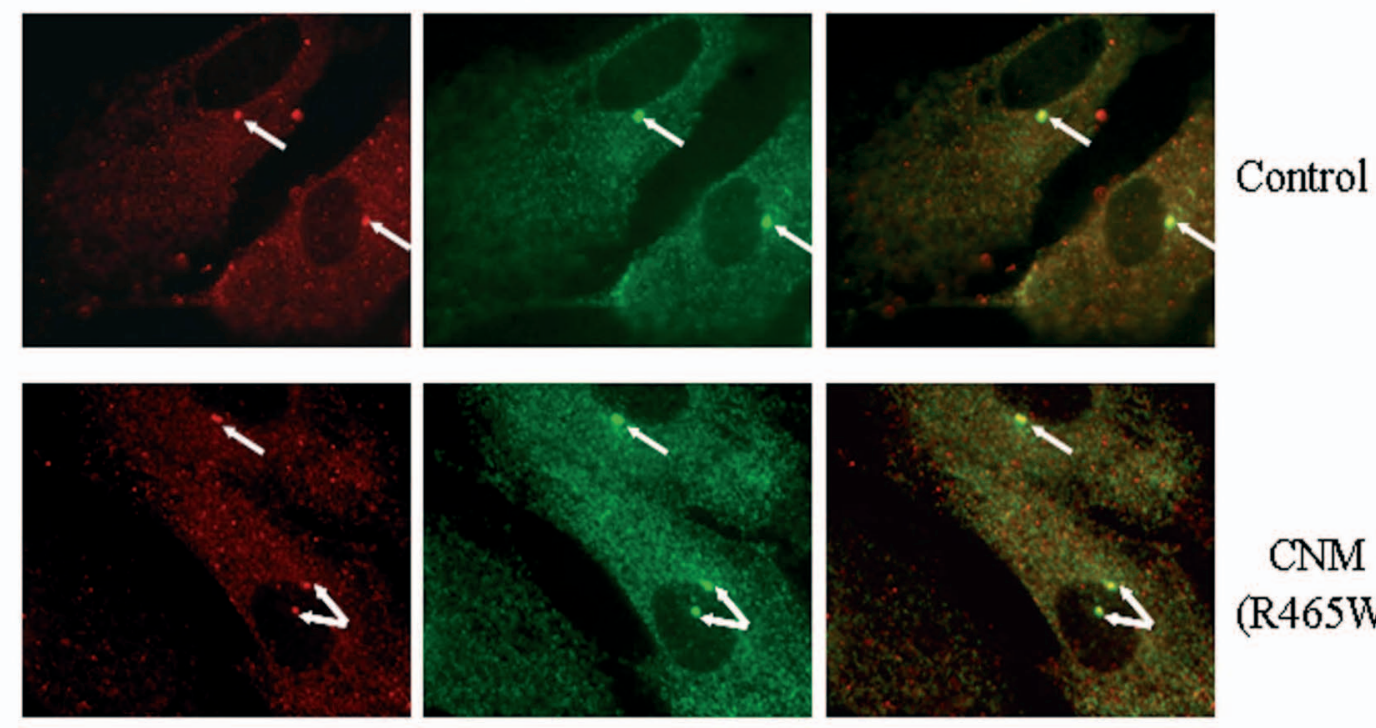

CNM

(R465W)

c Control $\mathrm{CNM}(\mathrm{R} 465 \mathrm{~W})$
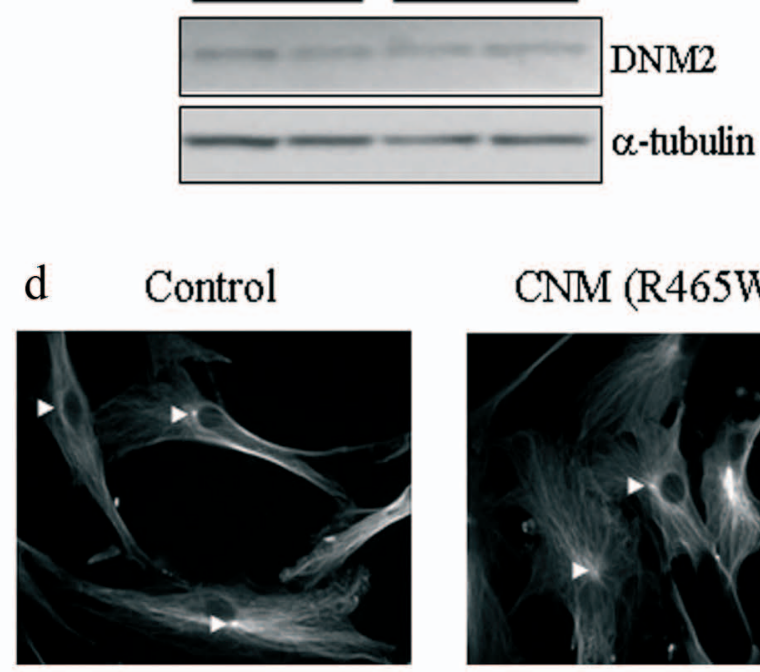

CNM (R465W)

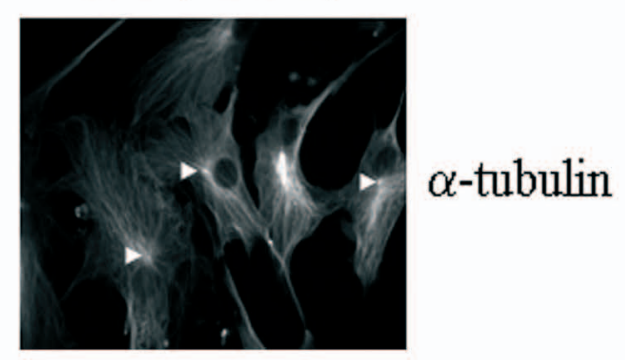




\begin{tabular}{|c|c|c|c|c|c|c|c|c|c|}
\hline \multirow[b]{2}{*}{ Marker } & \multicolumn{3}{|c|}{ Distance } & \multicolumn{5}{|c|}{$\theta$} & \multirow[b]{2}{*}{0.4} \\
\hline & cM & $\mathrm{Mb}$ & 0.0 & 0.01 & 0.05 & 0.1 & 0.2 & 0.3 & \\
\hline D19S216 & 20.01 & 4.9 & -4.75 & -3.60 & -1.93 & -1.04 & -0.27 & 0.03 & 0.10 \\
\hline D19S1034 & 20.75 & 6.06 & -0.26 & 0.51 & 1.10 & 1.25 & 1.14 & 0.79 & 0.36 \\
\hline D19S884 & 26.37 & 8.05 & 6.20 & 6.08 & 5.60 & 4.97 & 3.66 & 2.28 & 0.95 \\
\hline D19S865 & 32.39 & 9.03 & 4.38 & 4.29 & 3.96 & 3.52 & 2.60 & 1.62 & 0.69 \\
\hline D19S584 & 34.25 & 11.06 & 2.93 & 2.85 & 2.53 & 2.13 & 1.35 & 0.68 & 0.20 \\
\hline D19S226 & 42.28 & 14.49 & 3.98 & 3.87 & 3.41 & 2.83 & 1.71 & 0.81 & 0.26 \\
\hline D19S432 & 42.28 & 15.54 & 5.36 & 5.24 & 4.75 & 4.13 & 2.92 & 1.77 & 0.69 \\
\hline D19S899 & 45.48 & 17.09 & -0.87 & -0.40 & 0.31 & 0.59 & 0.66 & 0.48 & 0.23 \\
\hline
\end{tabular}

Supplementary Table 1. Cumulative two-point lod scores for chromosome 19p13 markers.

$\theta$ represent recombination fractions. For each marker, the genetic distance is given in centiMorgans according to the Genetic Map index from Marshfield database (http://research.marshfieldclinic.org), and the position in Megabases from the telomere according to the NCBI Map Viewer server (http://www.ncbi.nlm.nih.gov/mapview/). 


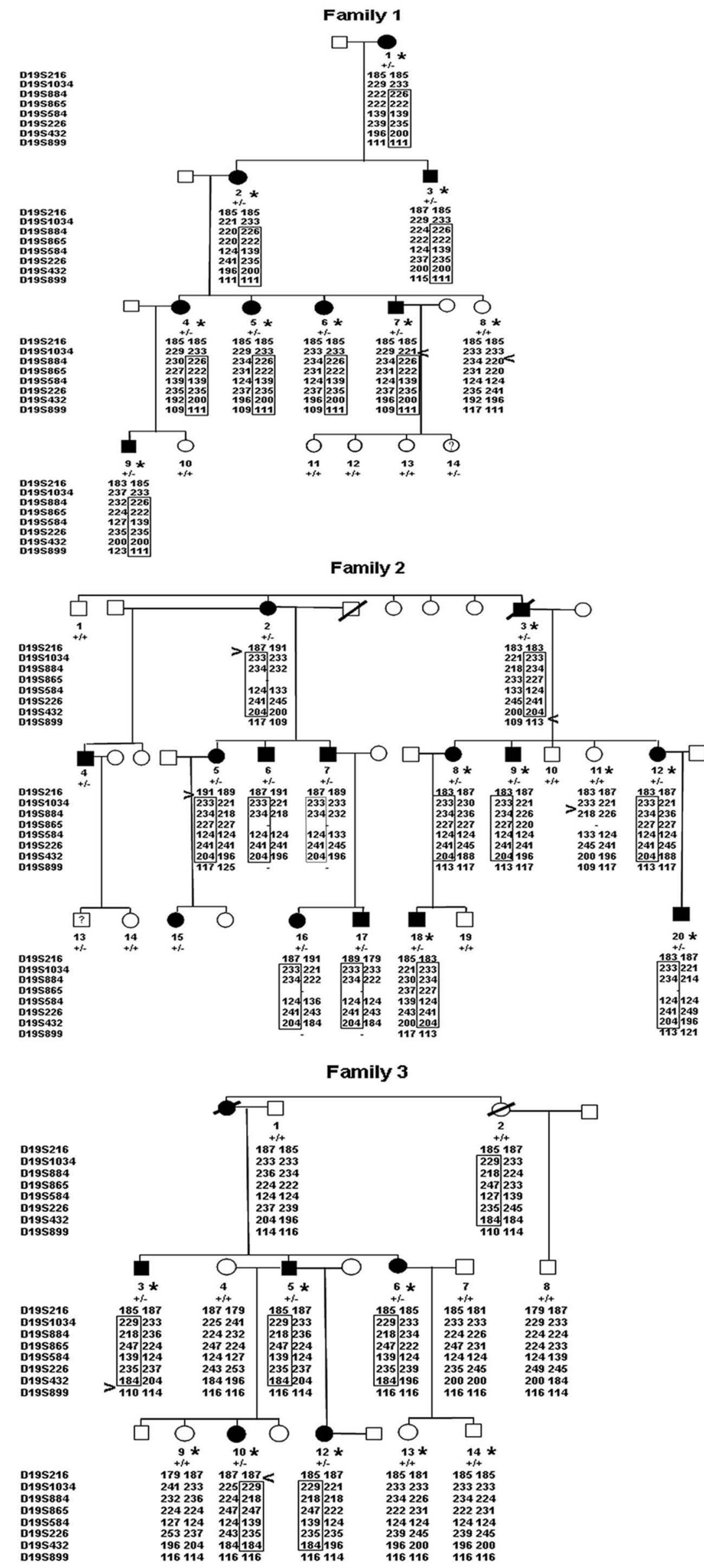

Supplementary Figure 1. Phenotype and haplotype analysis of 3 autosomal dominant CNM families and DNM2 genotyping.

The phenotype of CNM families is indicated using filled symbols (clinically affected subjects) and open symbols (unaffected subjects). Squares indicate males, circles indicate females and slashed-through symbols indicate deceased subjects. "?" indicates a young subject (3 year-old) with a mild ptosis (Subject 14 in Family 1) and a subject without clinical information (Subject 13 in Family 2). Asterisks show individuals initially included in the genome-wide screening. The haplotypes cosegregating with the disease are boxed for each family. < and > indicate the location of the recombination events. In Family 3 , the R369W mutation likely represents a de novo event in the affected grand-mother since her unaffected sister was carrier of the disease haplotype but not of the mutation, in contrast to all the other affected members of the family. For the DNM2 genotyping, $+/+$ and $+/$ - show homozygous wild type sequence and heterozygous mutated sequence, respectively. 


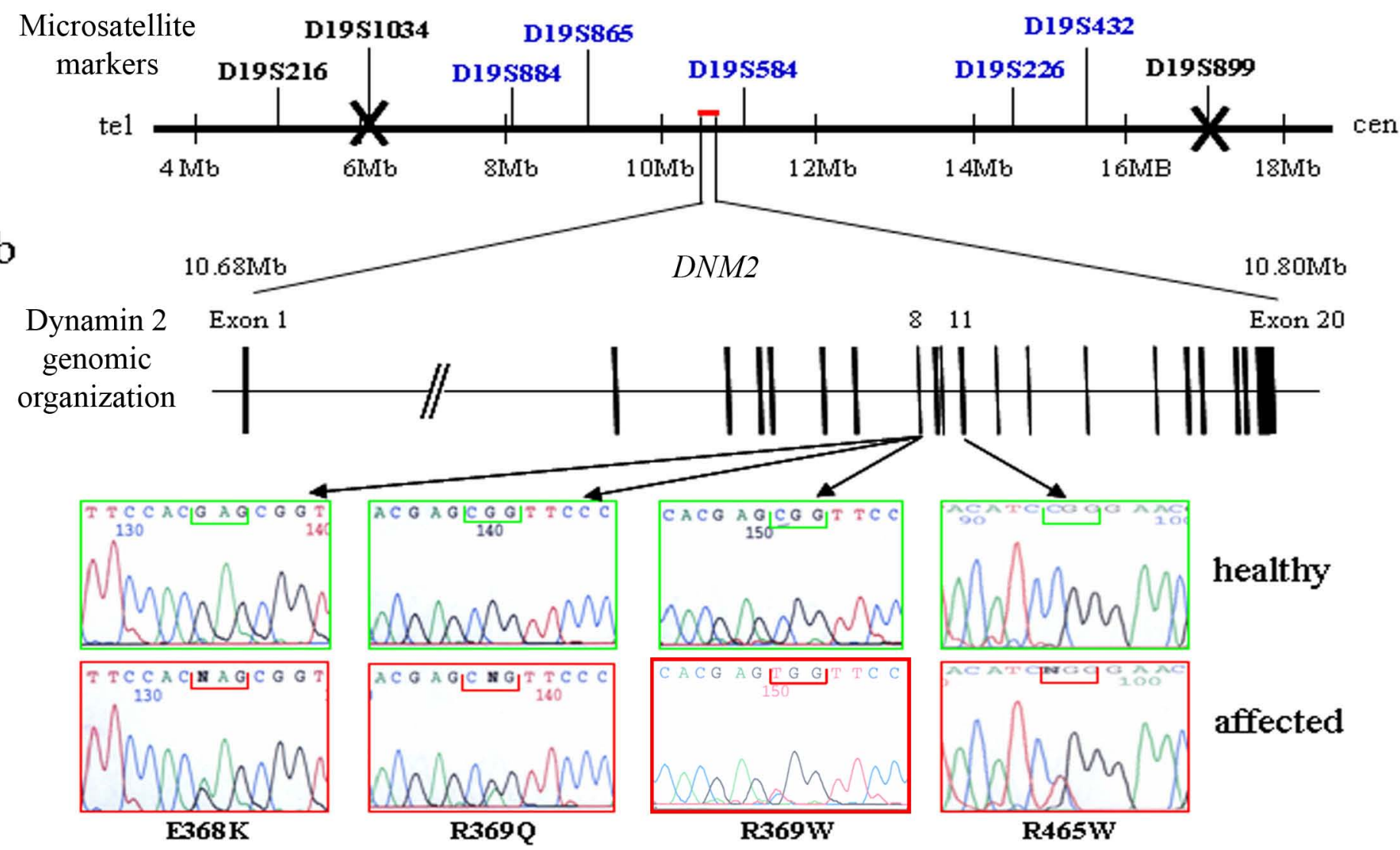

c
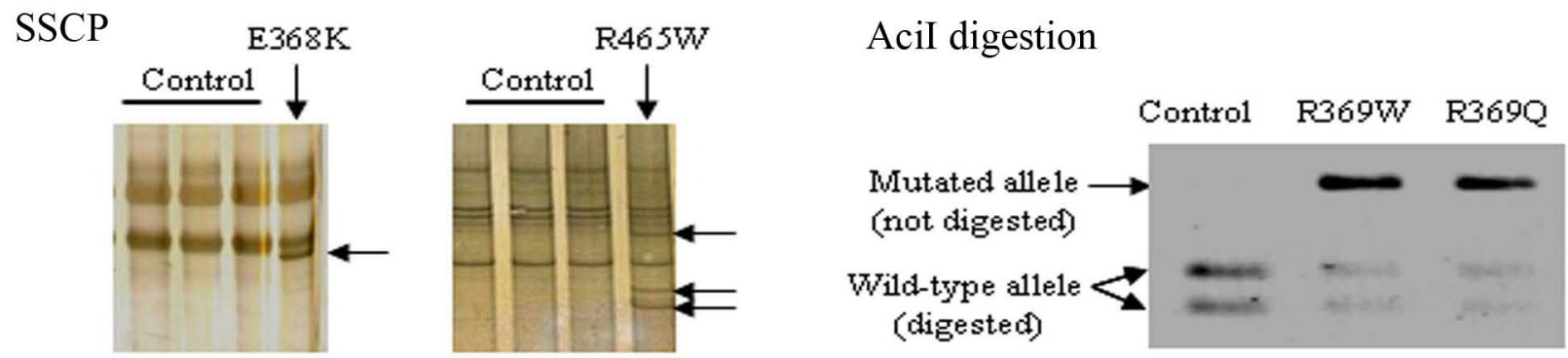

\section{Supplementary Figure 2. Physical map of the CNM locus and DNM2 mutations.}

(a) Physical map in the 19p13.2 genomic region and microsatellite markers used for linkage analysis. Linked markers with positive cumulative lod scores for Families 1 to 3 are indicated in blue and recombination events are indicated by a cross. tel: telomere. cen: centromere. (b) Genomic organization of DNM2 consisting of 20 coding exons and electrophoregrams of DNM2 exons 8 and 11 showing wild type sequence in unaffected subjects and the heterozygous mutations in affected subjects. Heterozygous mutations result in amino-acids changes located to position $368(\mathrm{G} \rightarrow \mathrm{A}$ that changes glutamic acid to lysine), position $369(\mathrm{C} \rightarrow \mathrm{T}$ that changes arginine to glutamine and $\mathrm{G} \rightarrow \mathrm{A}$ that changes arginine to tryptophane) and position $465(\mathrm{C} \rightarrow \mathrm{T}$ which changes arginine to tryptophane). (c) SSCP profiles of exon 8 and exon 11 and AciI restriction pattern of exon 8. Left: typical profiles obtained by SSCP for mutation E368K in exon 8 and R465W in exon 11 in comparison with PCR products from control subjects. Arrows indicate additional bands in the mutated subjects. Right: typical restriction pattern of exon 8-PCR products from control and mutated subjects, digested by AciI enzyme. Both mutations R369W and R369Q abolish restriction site of AciI and the undigested allele corresponding to the mutant form is identified on agarose gel. 


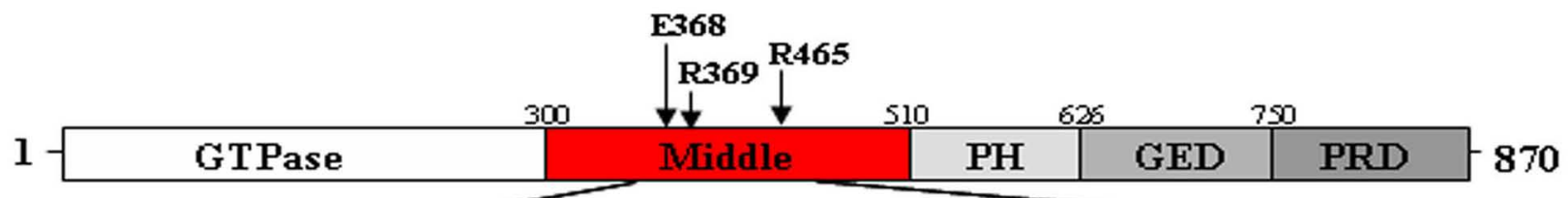

\begin{tabular}{|c|c|}
\hline \multirow[b]{2}{*}{ DYT2 HUMAN } & \multirow[b]{2}{*}{ BRTELFTP } \\
\hline & \\
\hline DYN2_MOUSE & 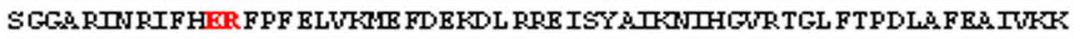 \\
\hline GN_FIJMAN & 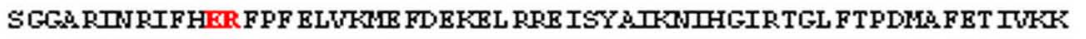 \\
\hline YN3_HUMMAN & KINRIF HER F PF ETVKHE FNEKEL RRE ISY A IKINIHGIRTGL FTPDM \\
\hline YN_Zebr & DNRIF HER F PF ELVKME FDEKEL RRE ISY A TRINIHGIR TGL F TPD \\
\hline YN_Dro & GGSKINRIF HER LRF EIUKMACDEKEL RRE ISF AIRNIHGIRUGL FTP \\
\hline YN_C. Eleg & GGA RTN RLF HER F PF EIVKME IDEKRM RKE IQY A IRNIHGIRVGL FT PDM \\
\hline \multirow[t]{2}{*}{ YN_Yeast } & CGGA RTYYTYNNU FGNSLKSID PTSNLSULDURT AIRNST GPRPTL FUP ELA FDL LVKP \\
\hline & 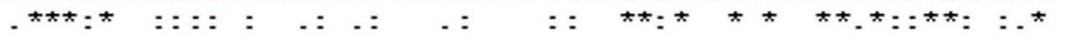 \\
\hline 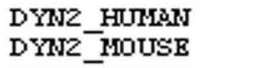 & FTERTITTY \\
\hline YNI_HUMAN & QUKKIRE PCLKCUDMU ISE LIS TVRQC-TKKLQQYPRLRE EME RIVT THIRE REGRTF \\
\hline YN3_HUMAN & QTUKLKGGPSLKSUDLV IQE LTNTUKKC- TKKLLWN F PRLCE ETE RTV ANHHIRE REGKTK \\
\hline YN_zebrafi & QTUKLKG PCLKCUDMU IQE LINTVQQC-THKLES F PKLRE ETE RTVT THIRE RESQTK \\
\hline DYN_Drosophila & QIAL LKE PUIKCUDLUUQE LSUUURMC-TAKMSRYPRLRE ET E RII T THURQ REHSCKK \\
\hline \multirow[t]{2}{*}{$\begin{array}{l}\text { DYN_C. Elegans } \\
\text { DYN_Yeast }\end{array}$} & QITRLKE PSLKCUDLUNE LWNUIRQC- ADTMSRYPRLRD ELE RTWUSHIR \\
\hline & $*: *:: * \ldots *$ \\
\hline
\end{tabular}

\section{Supplementary Figure 3. Predicted DNM2 structure and multiple protein alignment.}

The predicted protein structure includes a tripartite GTPase domain (GTPase), a middle domain (Middle), a Pleckstrin Homology domain (PH), a GTPase effector domain (GED) and a Proline rich domain (PRD). All mutations were detected in 3 amino-acids (368, 369 and 465) located in the middle domain of the DNM2 sequence and affect conserved residues within the human dynamin family and orthologues from different species (indicated in red). 


\section{Supplementary Methods}

CNM families. For identification of the CNM locus, three large autosomal dominant CNM families were selected: Families 1 and 3 with classical AD CNM form and Family 2 with a milder phenotype associated with diffuse muscle hypertrophy. CNM was diagnosed because several members of these 3 families presented typical clinical pictures of centronuclear myopathy (delayed milestones, muscle weakness, contractures, ptosis, ophtalmoparesis) coexisting with the following histopathological pattern of muscle biopsies: i) centrally located nuclei in $>5 \%$ of extrafusal fibres, ii) type 1 fibre hypotrophy evidenced through myosin adenosine triphosphatase (ATPase; $\mathrm{pH}$ 9.4) staining, and iii) radial arrangement of sarcoplasmic strands around central nuclei on nicotinamide adenosine-tetrazolium reductase staining. Patients were included in the linkage analysis with an affected status only if they present characteristic clinical signs of CNM, with or without biopsy examination. Thus, twenty affected patients with biopsy and six patients without biopsy (patient 7 in Family 1 and patients 5, 12, 16, 17 and 20 in Family 2) were included. In order to avoid inclusion of asymptomatic carriers as healthy in the Lod score calculation, subjects were included as healthy in the analysis only if their muscle biopsy was devoid of morphological abnormalities.

Linkage analysis. Blood samples were obtained after informed consent from eleven families with CNM affected patients and DNA was extracted according to standard procedures. A genome-wide screening was performed on Families 1 and 2 using a 400 microsatellite marker set (Applied Biosystems) at the "National Centre of Genotyping” (Evry, France). Genetic linkage of the disease was performed by analysis of the microsatellite markers D19S216, D19S884, D19S865, D19S226, and D19S899 from genome-wide screening and additional markers D19S1034, D19S584 and D19S432. Polymerase chain reactions (PCR) were carried out with 24 ng DNA. We used the touchdown PCR method, with annealing temperature decreasing from 70 to $60^{\circ} \mathrm{C}$ in the first 10 cycles, and fixed at $60^{\circ} \mathrm{C}$ in the final 25 cycles. All PCRs were done using Platinum Taq polymerase (Invitrogen, France) for amplification. PCR products were amplified using forward primers labelled with 6-Fam or Hex fluorochromes, and migrated on an ABI 377 automated sequencer. Data were analyzed by the Genotyper 2.5 software (Applied Biosystems) and haplotypes were constructed. Statistical analysis was performed using the LINKAGE 5.2 package assuming an autosomal dominant inheritance, an equal male and female recombination rate, a disease gene frequency of 0.0001 , and a 
penetrance of 0.9. Cumulative lod scores for Families 1 to 3 were calculated with isoallelic frequencies.

DNM2 sequencing. Six AD families (Families 5, 6, 7, 8, 9 and 11) and two families with sporadic cases (Families 4 and 10) were added to Families 1, 2 and 3 for the screening of the DNM2 gene mutations. The genomic sequence of the human dynamin 2 was used to design intronic primers to amplify the 20 exons. Primer sequences are available on request. Polymerase chain reactions (PCR) were carried out with 100 ng DNA. We used the touchdown PCR method, with annealing temperature decreasing from 70 to $60^{\circ} \mathrm{C}$ in the first 10 cycles, and fixed at $60^{\circ} \mathrm{C}$ in the final 30 cycles. All PCRs were done using Platinum Taq polymerase (Invitrogen, France) for amplification. Purified PCR products were analyzed in the both strands by uni-directional sequencing with ABI PRISM BigDye Terminator cycle sequencing ready reactions kit 3.1 and run on a 377 Genetic Analyzer (Applied Biosystems). Sequences were analyzed using Sequencing Analysis 3.4 software (Applied Biosystems). All detected mutations were analyzed at least twice by sequencing in both directions.

Validation of DNM2 mutations. Absence of mutations was checked in 150 healthy Caucasian control subjects of European origin. Controls were screened for mutations by Single Strand Conformation Polymorphism (SSCP) for mutations R465W and E368K or restriction enzyme digestion using AciI for mutations R369W and R369Q. For SSCP, exon 8and exon 11-PCR products were denatured $5 \mathrm{~min}$ at $94^{\circ} \mathrm{C}$. Samples were then submitted to electrophoresis on non-denaturing $10 \%$ polyacrylamide gels at $20^{\circ} \mathrm{C}$ for exon 8 or $25^{\circ} \mathrm{C}$ for exon 11, and subsequently analyzed through silver nitrate staining. For restriction pattern analysis, exon 8-PCR products were digested overnight at $37^{\circ} \mathrm{C}$ using $2 \mathrm{U}$ of AciI restriction enzyme (New England, BioLabs inc.). Digestion products were then submitted to electrophoresis on $2 \%$ agarose gels.

Cell culture, constructs and transfection. Fibroblasts were cultured from skin biopsies of a 33 year-old patient from Family 1 and a 28 year-old patient from Family 6 in Dulbecco's modified Eagle's medium (DMEM) supplemented with 10\% foetal calf serum (FCS) in a 5\% $\mathrm{CO}_{2}$ incubator at $37^{\circ} \mathrm{C}$. For transfection studies, skin fibroblasts from two healthy adult subjects were used as controls. The open reading frame of wild-type DNM2 and of two mutants (C1393T and C1105T corresponding to amino acid changes R465W and R369W, respectively) were generated by RT-PCR from lymphocyte mRNA and inserted in frame with 
the Green Fluorescent Protein (GFP) in pGFP-NT-TOPO-TA vector (Invitrogen, France). Human fibroblasts were seeded in $35 \mathrm{~mm}$ diameter plates and transfected at 50\% confluency using Lipofectamine reagent following manufacturer's instructions (Invitrogen, France). Briefly, $1 \mu$ g plasmid and $10 \mu \mathrm{l}$ Lipofectamine were mixed for $30 \mathrm{~min}$ in OptiMEM-1 culture medium (Invitrogen) and then added to cells for 4 hours at $37^{\circ} \mathrm{C}$. This transfection medium was then replaced by DMEM-10\% FCS for 2 days before $\gamma$-tubulin immunolabelling.

Immunocytochemistry. Fibroblasts were washed in PBS and then fixed 20 min at $-20^{\circ} \mathrm{C}$ in acetone before immunolabelling. Non specific sites were blocked in PBS with 5\% FCS and 0.01\% Triton X-100 for 90 min and incubated overnight with the mouse monoclonal antibody C11 directed against human $\gamma$-tubulin (Santa Cruz Biotechnology) or mouse monoclonal antibody against human $\alpha$-tubulin (Sigma Aldrich) diluted at 1:200 in blocking buffer. After washing, immunostaining was revealed by incubation for 2 hours with an anti-mouse antibody-Cy3 (Jackson ImmunoResearch) diluted at 1:200 in blocking buffer. For double immunolabelling, primary antibodies against human dynamin 2 (C18-goat antibody, Santa Cruz Biotechnology) and against $\gamma$-tubulin were mixed at 1:200 in blocking buffer. Immunostaining was revealed using a mixture of rabbit anti-goat-Cy3 and sheep anti-mouseFITC (Chemicon International) at 1:400 in blocking buffer. Labelled cells were visualized by fluorescence microscopy (Axiophot system Zeiss, Germany) coupled with a CCD camera and images were acquired using the Metaview software (Universal Imaging, USA).

Western-blotting. Twenty $\mu \mathrm{g}$ of protein from control and CNM-fibroblasts in loading buffer (50 mM Tris-HCl, 2\% SDS, 10\% glycerol, 1\% $\beta$-mercaptoethanol and bromophenol blue) were submitted to electrophoresis on a 10\% SDS-PAGE gel and then transferred onto polyvinylidene difluoride (PVDF) membranes (Invitrogen). Non specific sites were blocked for 2 hours at room temperature in PBS with 5\% nonfat dry milk and $0.1 \%$ Triton X-100. Incubation with primary antibody was performed overnight at $4{ }^{\circ} \mathrm{C}$ for antibody against DNM2 (C18-goat polyclonal antibody raised against a peptide mapping at the carboxy terminus of DNM2, Santa Cruz Biotechnology, 1:200) or 1 hour at room temperature for antibody against $\alpha$-tubulin (mouse monoclonal antibody from Sigma Aldrich, 1:1000) diluted in the blocking buffer. After washing, membranes were incubated 2 hours at room temperature with Horseradish Peroxidase (HRP)-conjugated secondary antibodies (anti-goat-HRP from Jackson ImmunoResearch and anti-mouse-HRP from Dako) diluted at 1:2000 in blocking 
buffer. Detection was performed using the Supersignal West Pico Chemiluminescent kit (Pierce).

Accession numbers. Human DNM2 mRNA GenBank NM_001005360. Human DNM2 genomic sequence GenBank NT_011295. Human DNM2 protein Genbank P50570. Mouse DNM2 protein Genbank P39054. Human DNM1 protein Genbank Q05193. Human DNM3 protein Genbank Q9UQ16. Zebrafish DNM protein Genbank AAH65325. Drosophila DNM protein Genbank P27619. Caenorhabditis elegans DNM protein Genbank P39055. Yeast DNM protein Genbank P54861. 